\title{
Sensorless Landing Control Strategy of Bistable Permanent Magnet Actuator
}

\author{
Bo Li, ${ }^{1}$ Cao Tan $\left(D,{ }^{1}\right.$ Geng Wang, ${ }^{2}$ Wenqing Ge, ${ }^{1}$ and Binbin Sun ${ }^{1}$ \\ ${ }^{1}$ School of Transportation and Vehicle Engineering, Shandong University of Technology, Zibo 255000, China \\ ${ }^{2}$ State Key Laboratory of Structural Analysis for Industrial Equipment, Dalian University of Technology, Dalian 116024, China \\ Correspondence should be addressed to Cao Tan; njusttancao@yeah.net
}

Received 31 March 2019; Accepted 17 April 2019; Published 11 June 2019

Academic Editor: Filippo Cacace

Copyright (c) 2019 Bo Li et al. This is an open access article distributed under the Creative Commons Attribution License, which permits unrestricted use, distribution, and reproduction in any medium, provided the original work is properly cited.

\begin{abstract}
Bistable permanent magnet actuator (BPMA) has been widely used in on/off application. However, the response time and landing performance of on/off actuator are contradictory. Through analyzing the landing control goal of on/off actuator and sensorless technology features of the BPMA, a new sensorless landing control strategy was proposed using armature position detection instead of real-time displacement estimation or displacement sensor. The feasibility and effectiveness of the method are verified by experiment and simulation, the operation quality of BPMA is effectively improved. The results show that the first bounce decreases by $85.7 \%$ and the second bounce is avoided when the iteration converged, compared to the condition without landing control, while the response time increased by $6.8 \%$. Meanwhile, the landing velocity decreased more than $50 \%$ and sound intensity at different frequencies reduced more than $4 \mathrm{~dB}$ with sensorless landing control strategy. Although in this paper the control strategy was employed to a BPMA, its structure can be used to analyze and design a wide range of on/off actuator.
\end{abstract}

\section{Introduction}

As a kind of energy converter, BPMA has been widely used in applications ranging from automation to transportation because of avoiding unacceptable weakening performance and reducing power consumption resulting from holding current [1-3]. However, the undesired impact issues of bounce and noise dramatically reduce the durability, reliability, and precision of the BPMA $[4,5]$.

Up to date, there are two main methods for actuators to achieve self-holding technology: mechanical self-holding and permanent magnetic self-holding. The mechanical selfholding mode makes the dynamic mass increase. However, the mechanical self-holding is not beneficial to actuator's driving force [6,7]. BPMA is an actuator towards a reduction in the power consumption or improvement the fast response, through advances in high-energy permanent magnet (PM) materials like neodymium-iron-boron $(\mathrm{NdFeB})$ [8]. But the BPMA possesses quite nonlinear force/stroke characteristic, which results in insufficient driving force when mover is away from target end $[9,10]$. Spring mechanism was introduced to solve this problem, which makes the actuator more complex and impairs holding force. In previous work, the holding force, start-up force and force/stroke characteristic of the BPMA are markedly increased [11]. However, as for the BPMA without spring mechanism, new challenges are brought in landing control because of holding force.

Typically, soft-landing control algorithms were functioned to limit the valve seating velocity, which mainly include iterative learning control [12], inverse system method control [13], and sliding mode control [14, 15]. As for BPMA landing control, ensuring fast response simultaneously is the major challenge. It is different from the traditional softlanding control of electromagnetic actuators. Much more sensor-based and senseless soft-landing control have been discussed, but little study on BPMA landing control has been reported. Moreover, with the actuator being used for sensing purposes the external transducer and its accessories, such as mechanical layout and cabling, can be saved. This results in considerably reduced system cost and in improved robustness which have paramount importance. Hence, [16] used an electric brake reducing the speed at the end of the stroke for circuit breaker. The electric brake needs an additional brake which increases the system volume. More 


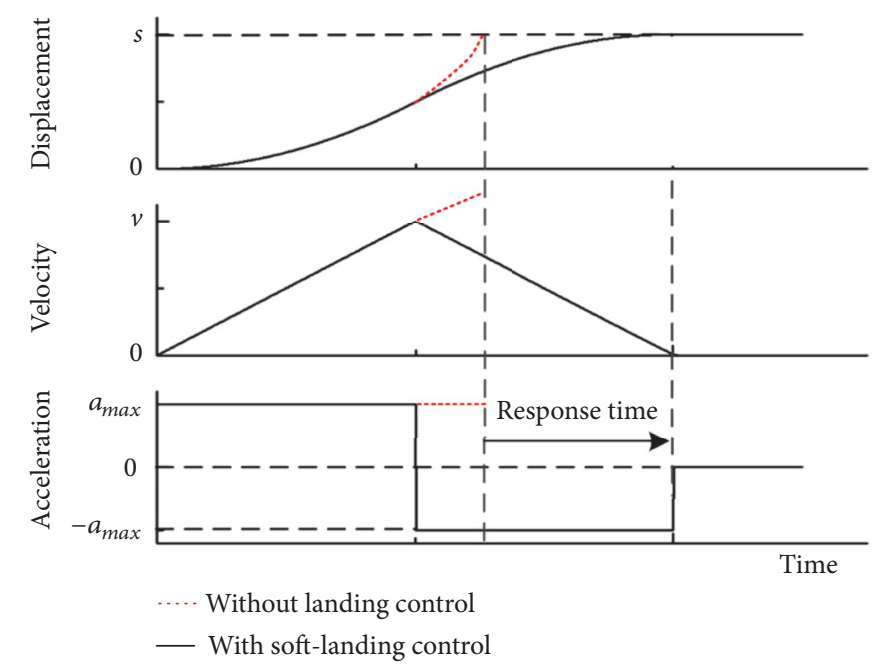

FIgURE 1: The landing process with and without landing control.

general, there are three methods to realize sensorless control. References [17-19] presented sensorless methods of position or velocity detection using magnetic signal and electric signal to realize senseless control. Meanwhile, input signal methods of position detection have been discussed [20, 21]. Moreover, sliding mode [22], nonlinear observer, Kalman filters [23], and hysteresis hybrid extended Kalman filter have been proposed for sensorless control [24]. The methods are restricted in many application fields, due to the algorithm being hard to implement.

This paper is organized as follows. In Section 2, the landing control problem of BPMA is analyzed in detail. Section 3 proposes a sensorless landing control strategy for BPMA. The sensorless landing control strategy is verified and analyzed in Section 4. This paper is concluded in Section 5.

\section{Problem Analysis}

2.1. Problem Description. The landing performance of actuator is the concentrated reflection of the dynamic operation process, which has the main influence on the reliability and durability of the electromechanical system. As for on/off actuator, the response time of on/off action is also an important factor affecting the performance of the system. However, the response time and landing performance of actuator are contradictory. The landing process of armature without landing control and with ideal soft-landing control is shown in Figure 1.

The armature without landing control keeps fast response with maximum acceleration, which leads to intense impact in landing process. In the ideal soft-landing control process, the armature accelerates to the midpoint of stroke with the maximum acceleration and then decelerates to the endpoint with the maximum acceleration to make velocity and acceleration equal to zero. However, the actual soft-landing control requires the landing velocity to be less than a certain value, because of the electromechanical time constant. Balancing the influence on response time and intense impact is not only an important goal of landing control for on/off actuator, but also a big difference between the landing control strategy proposed in this paper and other strategies.

Besides, BPMA has been widely used as on/off actuator. The holding force is produced to eliminate holding current under the action of permanent magnetic polarization magnetic field. In order to produce electromagnetic force opposite to the holding force, excitation electromagnetic field must overcome the impact of permanent magnetic polarization magnetic field firstly. Therefore, many new problems are brought because of the holding force caused by permanent magnet. A BPMA without spring was optimized based on 3D finite element analysis in our previous work [11]. The BPMA is taken for example in this paper, which has cylindrically symmetrical configuration and consists of a coil, a coil framework, an armature, a sleeve, two permanent magnets, and an outer stator including yoke, pole shoe, and cover, shown as Figure 2.

Additionally, as shown in Figure 3, the iron loss, copper loss, and other losses account for $48.6 \%, 36.5 \%$, and $1.9 \%$ of total energy consumption in work cycles, separately. But landing dissipation energy (3.4J) accounts for $13 \%$ of total energy consumption in work cycles without load, which leads to serious energy waste and impact. Hence, an appropriate landing control strategy is essential for BPMAs.

2.2. Modeling and Validation. The BPMA employs the concept of magnetic field interaction generated by a stationary coil and permanent magnets. The mechanical model can be represented by Newton's second law as

$$
m \frac{d^{2} x}{d t^{2}}=F_{m}-c \frac{d x}{d t}-F_{\text {load }}
$$

where $m$ is the motion mass, $F_{m}$ is the electromagnetic force, $F_{\text {load }}$ is load, and the damping coefficient $c$ is $20 \mathrm{~N} /(\mathrm{m} / \mathrm{s})$. By making use of Kirchhoff's second law and Faraday's law, a differential equation relating the control coil voltage $(u)$, the coil current $(i)$, the coil resistance $(r)$, and the magnetic flux linkage $(\Psi)$ may be expressed as 


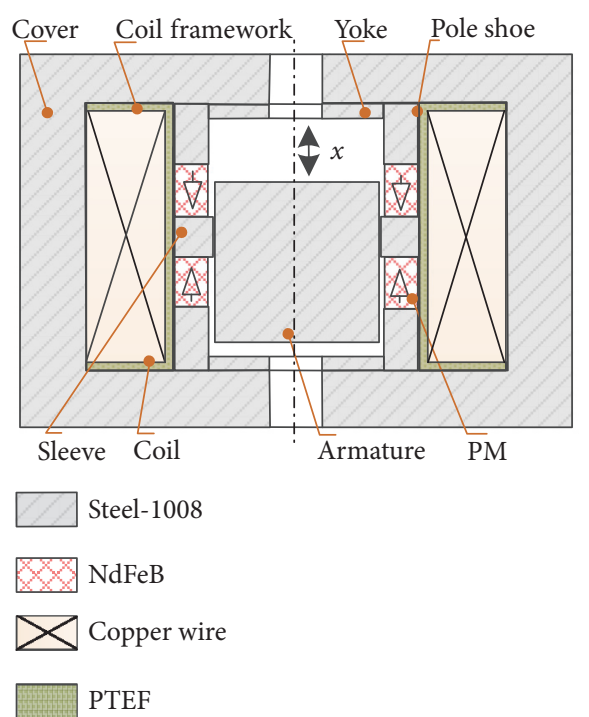

FIGURE 2: The schematic of BPMA.

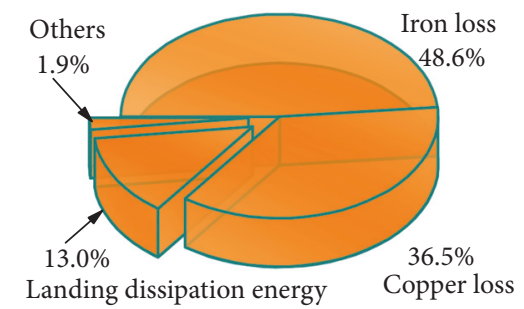

FIgURE 3: The cycle energy consumption of the BPMA.

$$
u=i r+\frac{d \psi(x, i)}{d t}=i r+L \frac{d i}{d t}+i \frac{d L}{d t}
$$

where $u, i, L, r$ are electrical parameters of winding expressed as voltage, current, inductance, and resistance, respectively. Note that, $F_{m}$ and $\Psi$ are obtained by finite element analysis.

A control-oriented dynamics mathematical model of actuator is established. The model is verified by the test bench proposed in $[5,11]$. The comparison between the simulation and experimental results with the same control signal are shown in Figure 4.

The response of BPMA is divided into two stages. In the first trigger phase, with current rapidly rising, the electromagnetic field reserves enough energy to overcome the load, and the armature has not yet started motion. In the second moving phase, the armature is away from the end of stroke, until it reaches another end of stroke. As shown in Figure 4, the simulation results are in good agreement with the experimental results. The control-oriented dynamics mathematical model can correctly reflect the changing rules of the parameters and meet the requirements of the simulation in control system.

\section{Sensorless Landing Control}

3.1. Analysis of Sensorless Technology. Considering the inductance of BPMA varies greatly with the armature displacement
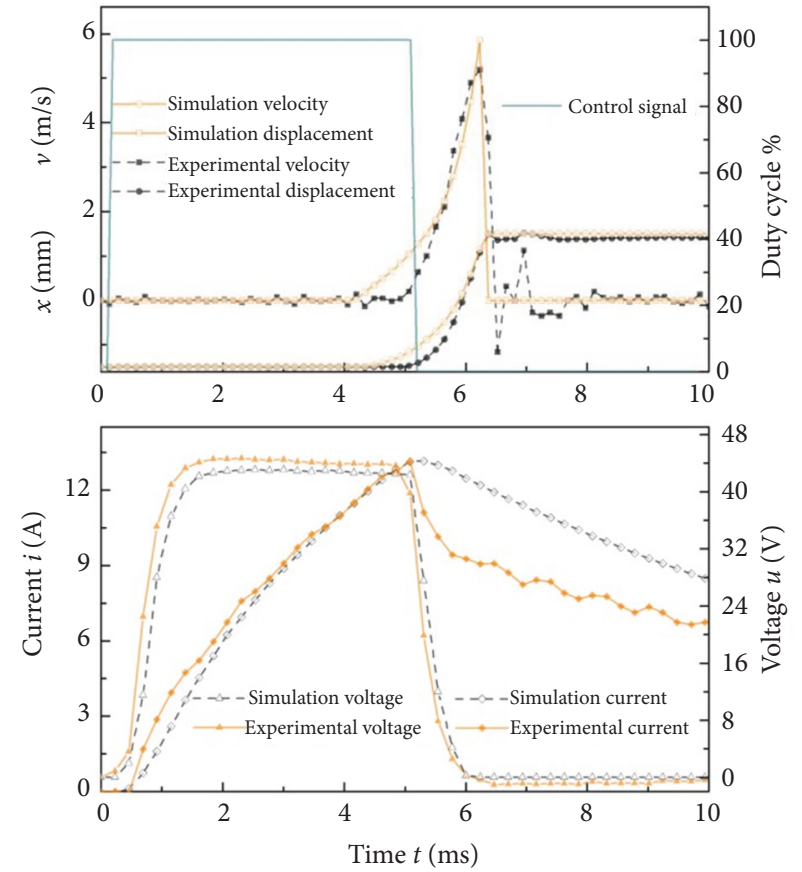

Figure 4: Comparison between the simulation and experimental results.

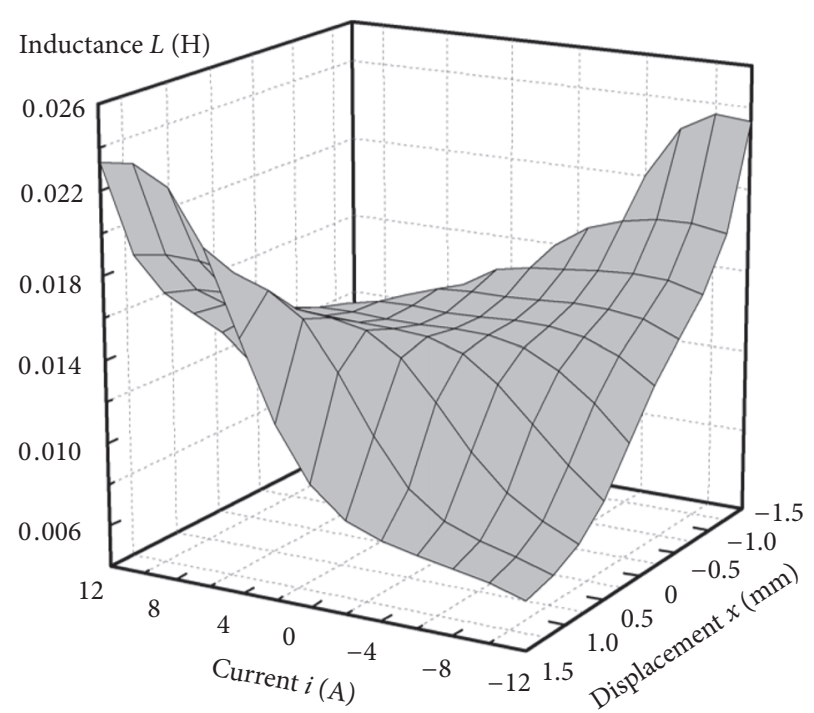

FIGURE 5: Relationship among current, armature displacement, and inductance of BPMA.

and winding current, shown in Figure 5 using Finite Element Analysis (FEA), the displacement of armature can be obtained through the corresponding relationship among inductance, winding current, and armature displacement. Finite Element Analysis for the BPMA is detailed description in our previous work $[5,11]$.

Inductance and current are electrical parameters related to winding and the equivalent circuit model is shown as in (2). The inductance is a function of armature position $x$ 


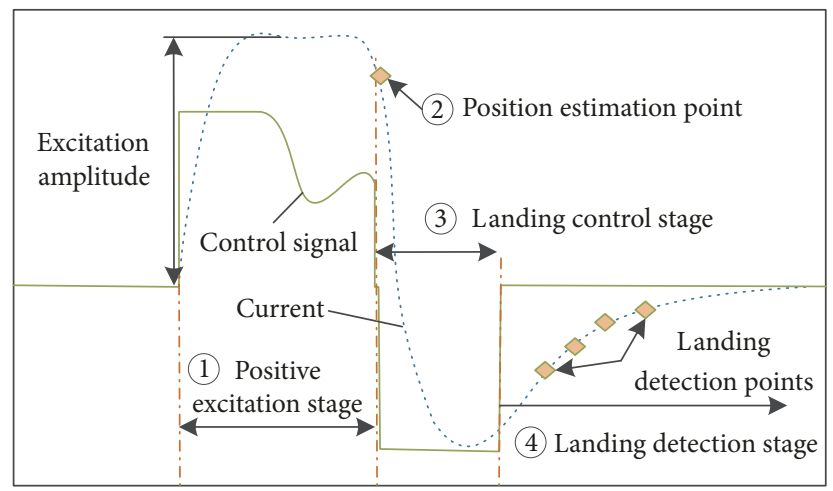

FIGURE 6: Schematic of proposed sensorless landing control strategy.

and current $i$, so the formula above can be further obtained as

$$
u=i r+\left[L(x, i)+\mathrm{i} \frac{\partial L(x, i)}{\partial i}\right] \frac{d i}{d t}+i \frac{\partial L(x, i)}{\partial x} \frac{d x}{d t}
$$

The third term on the right of the equation is motional electromotive force, which can be neglected on the conditions when the armature is static or when the research time is short enough not to make armature operate, or when the speed of armature is less than $1.5 \mathrm{~mm} / \mathrm{min}$ [17-19, 22-24]. It can be concluded that since the research time for control period discussed in this paper is $0.05 \mathrm{~ms}$, which is much shorter than mechanical time constant, there is no movement on armature. Then (3) can be simplified as

$$
u=i r+L_{e q}(x, i) \frac{d i}{d t}
$$

where $L_{e q}$ is total equivalent inductance considered iron loss. Then (4) can be expressed in another form (5), when the current change rate $\left(R_{c}\right)$ is not zero.

$$
L_{e q}(x, i)=(u-i r) \cdot\left(\frac{d i}{d t}\right)^{-1}
$$

Obviously, (5) is nontrivial if and only if $d i / d t \neq 0$. This condition is generally troublesome when the current is nearly a constant. In our experimental setup, a digital amplifier with a PWM-controlled MOSFET is used. Though the armature has reached its stable position, the current is not a constant and its derivative $d i / d t$ is nonzero. According to (4), when the excitation voltage and current value are known, the current change rate of the actuator is proportional to the total equivalent inductance and the armature position.

3.2. Establishment of Sensorless Landing Strategy. An energy distribution landing control algorithm was proposed. The main idea behind the approach is to distribute the overall mechanical energy of the BPMA's mechanical system. Assuming that the load driven by the actuator remains constant during the motion, the kinetic energy of the armature at landing point is the difference between the positive work done by electromagnetic force $\left(W_{F m+}\right)$ and the negative work done by electromagnetic force $\left(W_{\mathrm{Fm}_{-}}\right)$and load $\left(W_{\mathrm{Fm}-}\right)$ during the motion.

$$
\frac{1}{2} m v_{\text {landing }}^{2}=W_{F m+}-W_{F m-}-W_{F-}
$$

The above expression confirms that the energy can be directly controlled by means of $F_{m}$ (the coil current) being fed to the system. Thus, the proposed control is to distribute the energy according to the distribution positive and negative works done by $F_{m}$. Combined with the landing control goal and sensorless technology features of the BPMA, a new sensorless landing control strategy is proposed as shown in Figure 6.

The landing control process is divided into four stages within each switching. In the first positive excitation stage $\left(t_{p}\right)$, the excitation current is controlled to provide enough energy for the armature moving. The position estimation is done in the second stage $\left(t_{e}\right)$ within one cycle of the control clock. The voltage control signal is 0 in the second stage. The information of the estimation point includes $R_{c}$ and $t_{p} . R_{c}$ in position estimation point is controlled using the trigger point current rate shown in Figure 3, and then $t_{p}$ varies with the load. According to the load, different voltage control signal is applied in the landing control stage $\left(t_{l}\right)$. In the landing detection stage $\left(t_{d}\right)$, some landing estimation points are used to detection if the armature was landing by observing $R_{c}$, until the next control process begins. The sensorless landing control process is shown in Figure 7.

In the control process, the control signal $u_{p}$ is obtained from current controller and the control target is $i_{\text {max }}$. In order to reduce the control variables, the absolute value of $u_{l}$ is equal to $u_{\max }$. Therefore, the control variables needing further consideration from estimation point information are $t_{p}$ and $t_{l}$. In the next sections, the positive excitation controller and landing excitation controller are designed for $t_{p}$ and $t_{l}$, separately. $t_{l}$ works in this cycle, while $t_{p}$ works in the next cycle. Hence, the control strategy can quickly respond to the changes of load. Since the actuator on-off works in a similar way, only half of the cycle is described in the control process.

In addition, landing or not is an important basis to ensure the reliability of performance. According to Figure 5, inductance value is significantly different when the armature located at different endpoints of stroke and the current is the same. Using the experimental setup in $[5,11]$, the 


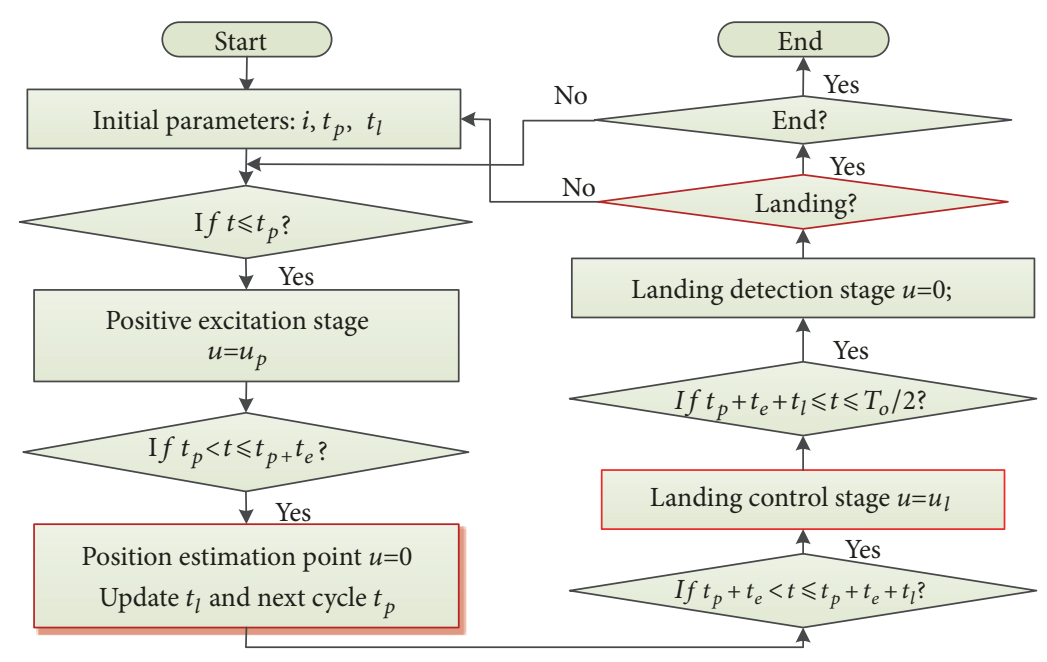

FIGURE 7: Sensorless landing control process.

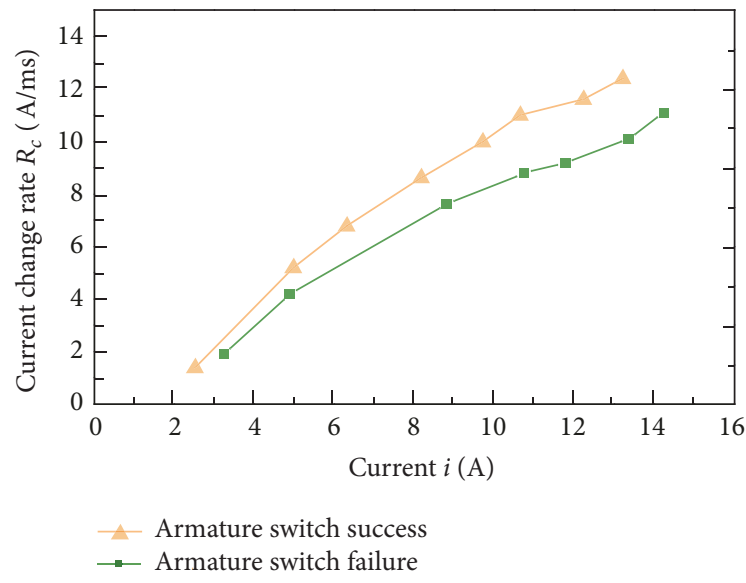

FIGURE 8: Current and corresponding current change rate at different condition.

test results show that $R_{c}$ with different current displays monotonically increasing function, shown in Figure 8, and the trend is more obvious by the increase of current. In landing detection points, the armature position is detected by analyzing the current and corresponding current change rate after the landing control stage. The sensorless landing control strategy using armature position detection instead of realtime displacement detection, which ensures the feasibility and practicability for on-off actuator landing control.

3.3. Positive Excitation Controller. The excitation current is controlled in the positive excitation stage, so that the current at the end of positive excitation stage is maintained as control target. At the position estimation point, the threedimensional relationship among inductance, winding current, and armature displacement of BPMA, shown in Figure 5, changes to two-dimensional relationship between inductance and armature displacement. Using the experimental setup in $[5,11]$, the relationship between $R_{c}$ and armature position with different $t_{p}$ is shown in Figure 9.
At the position estimation point, if switch was not done in positive excitation stage, the current change rate and armature position are one-to-one. And when $t_{p}$ is smaller than $4.2 \mathrm{~ms}$, the BPMA switches failed. The trigger point is the moment that armature just starts moving. The trigger point corresponds to a certain current change rate $\left(R_{c t}\right)$. Meanwhile, the coupling physical fields interactions in trigger and moving are illustrated in [5]; the trigger point moment $\left(t_{t}\right)$ reflects the load information. The relationship between $t_{t}$ and load is obtained from simulation. Due to the armature moves within milliseconds, $t_{t}$ is selected as target positive excitation time to leave more adjustable time for landing control.

Meanwhile, a positive excitation controller based on Iterative Learning Control (ILC) is proposed to control the position estimation point which becomes trigger point. The feedback signal is current change rate from current sensor and digital controller instead of displacement sensor. $R_{c t}$ is recorded as $y_{d}$; the error $e_{n}$ in $\mathrm{n}$-th cycle can be expressed as

$$
e_{n}=y_{d}-y_{n}
$$

The output of the next cycle is modified according to the error $e_{n}$ and the $\mathrm{n}$-th cycle output. The open-loop PD-type ILC is chosen, and the control law can be expressed as

$$
u_{n+1}=u_{n}+k_{p} e_{n}+k_{d} \dot{e}_{n}
$$

where $k_{p}, k_{d}$ are gain coefficient. After convergence, $t_{p}$ is controlled the actuator can trigger reliably with different load, which makes actuation adaptive to various loads.

3.4. Landing Excitation Controller. The landing dissipation energy is $3.4 \mathrm{~J}$ without landing control under no load condition. If $u_{l}$ is equal to $-u_{\max }$ when armature is moving, the landing dissipation energy is $0.6 \mathrm{~J}$ under no load condition. The landing dissipation energy $0.6 \mathrm{~J}$ corresponds to landing velocity $3.0 \mathrm{~m} / \mathrm{s}$. Therefore, the minimum landing velocity obtainable by the actuator is $3.0 \mathrm{~m} / \mathrm{s}$. In order to maintain the 


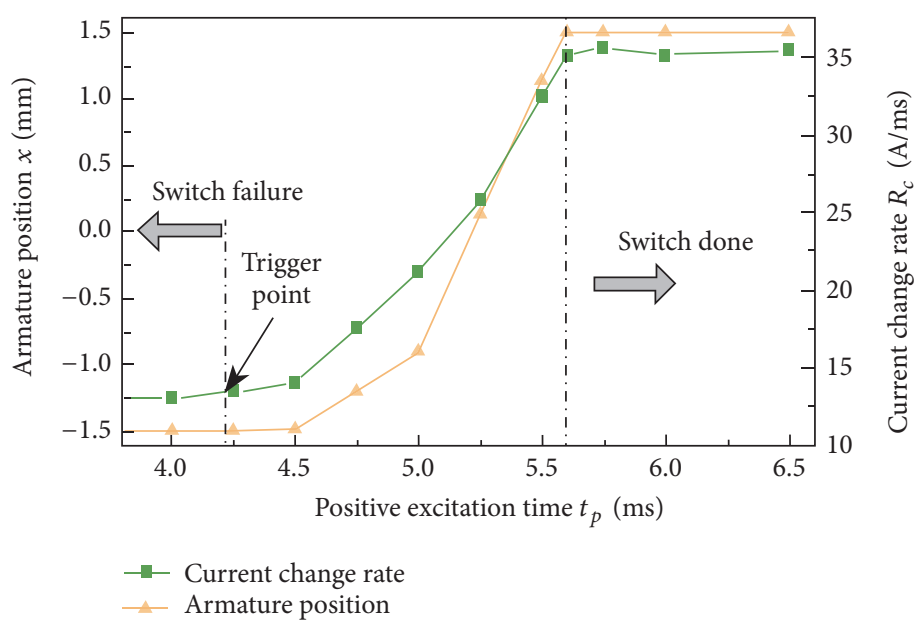

FIGURE 9: Relationship between current change rate and armature position.

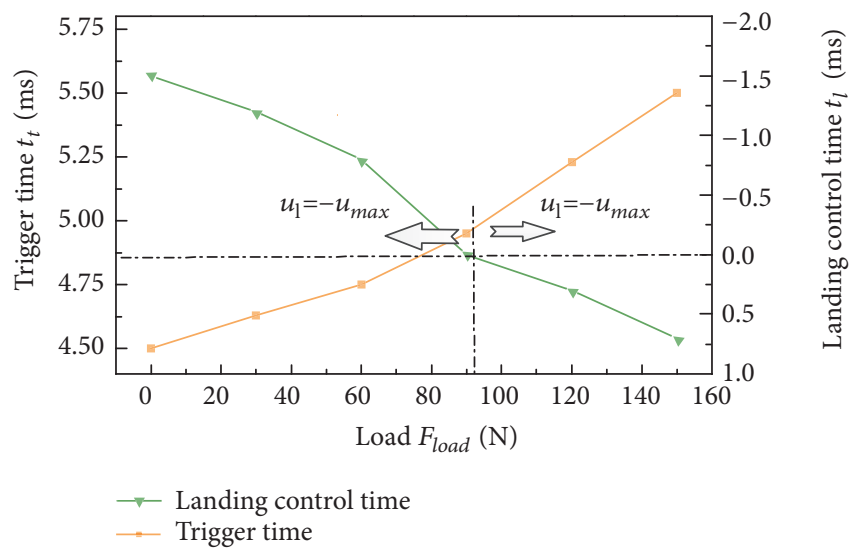

FigURE 10: Relationship among trigger time, landing control time, and load.

characteristics of fast response and keep the landing velocity consistent under different loads, the target landing velocity of the armature is set to $3.0 \mathrm{~m} / \mathrm{s}$. The load is identified according to the trigger time, and the corresponding excitation used in the landing control stage is obtained from load. The relationship among $t_{t}, t_{l}$ and load is obtained from simulation, where the minus sign represents the excitation in the opposite direction of motion, shown in Figure 10.

The maximum load of the system design is $150 \mathrm{~N}$. The relationship between $t_{t}$ and load is approximately linear. It is assumed that the load remains constant during the motion, due to the armature moves within milliseconds. When the load is $93 \mathrm{~N}$, the landing control time is 0 , which means the control signal is 0 in the landing control stage. When the load is smaller than $93 \mathrm{~N}$, the landing control time is smaller than 0 , which means the control signal is $-u_{\max }$ in the landing control stage. When the load is bigger than $93 \mathrm{~N}$, the landing control time is bigger than 0 , which means the control signal is $u_{\max }$ in the landing control stage. The bigger the difference between the load and $93 \mathrm{~N}$, the longer the landing control time is needed. The landing control time is adjusted within the current working cycle, which improves the realtime performance of the landing control of the actuator.

\section{Results and Discussion}

4.1. Variable Load Analysis. Combined with the above description, the landing control simulation model of BPMA is established based on MATLAB. The 100 work cycles with initial with $6 \mathrm{~ms}$ positive excitation time and $2 \mathrm{~ms}$ landing control time are simulated. The load is $50 \mathrm{~N}$ in the first 50 cycles, $10 \mathrm{~N}$ at the beginning of cycle 51 , and $120 \mathrm{~N}$ at the beginning of cycle 71 . The analysis results are shown in Figure 11 .

The BPMA can operate normally under the action of initial control parameters. Due to the positive excitation controller based on ILC, $t_{p}$ gradually converges to the target trigger time value under $50 \mathrm{~N}$ load and the excitation of $t_{l}$ changes to the corresponding value within 4 work cycles. Then the control system works stably, until the load is adjusted from $50 \mathrm{~N}$ to $10 \mathrm{~N}$. When the load becomes small, the control parameters are adjusted through iterative learning. In the adjustment process, the positive excitation time is longer than the convergence value of the target trigger time, while the landing control time is smaller than the corresponding required value under current load. The actuator operates normally, while the landing velocity increases sharply in the 


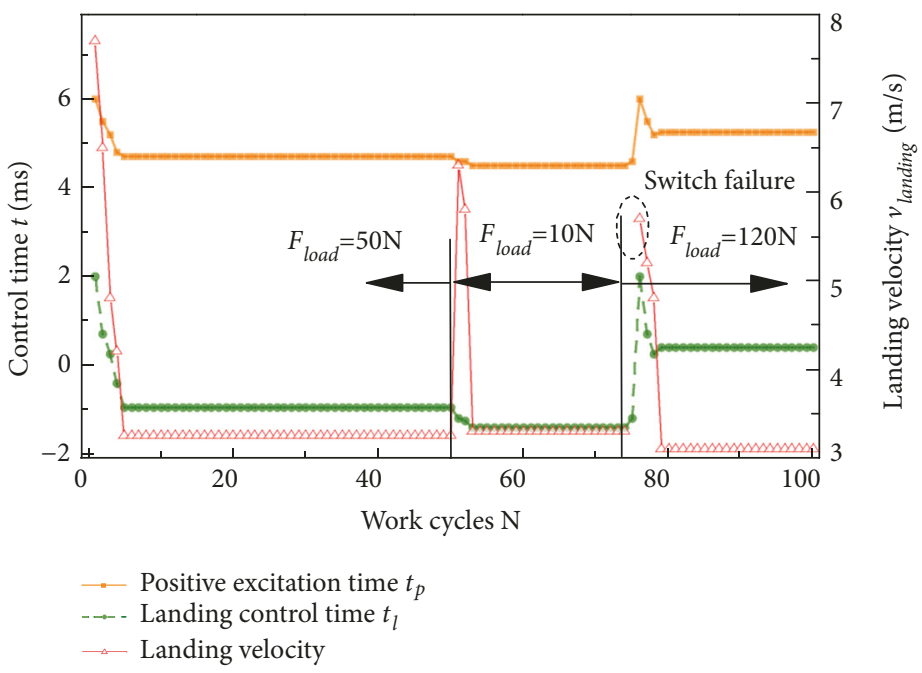

FIGURE 11: 100 work cycles simulation result with variable load.

first cycle after the change of load. And then the landing velocity gradually decreases with the change of the positive excitation period. The positive excitation time converges after three cycles, and the landing control time and the landing velocity also converge.

The BPMA does not function properly in the first cycle after the load changes from $10 \mathrm{~N}$ to $120 \mathrm{~N}$, due to $t_{p}$ being smaller than needed. Because of the actuator switch failure, the system initializes the control parameters. And then the positive excitation time converges after three cycles. When the control parameters converged, the actuator works stably and landing velocity is controlled near the target value, which verifies the feasibility and stability of the sensorless landing control strategy under variable load.

4.2. No Load Analysis. A 200-cycle successional test based on the test bench shown in [5] is carried out to verify the effect of sensorless landing control without load. Due to the limitation of test conditions, the test under load will be studied in the future. In addition, decibel meter with the test scope of 30$130 \mathrm{~dB}$ is used to test the working noise. The distance between the microphone and BPMA is fixed at $10 \mathrm{~cm}$, while the decibel meter is coaxial with BPMA. The successional test results of BPMA under sensorless landing control are shown in Figure 12.

The test results in the fact that, after 6 cycles of iteration, the current change rate of the position estimation point is stable around the target current change rate $\left(R_{t t}\right)$. The positive excitation time, landing control time, and landing velocity converge after 6 cycles of iteration. When the control parameters converged, the position estimation point is kept between $-1.45 \mathrm{~mm}$ and $-1.5 \mathrm{~mm}$. It is proved that the armature position estimation and positive excitation time controller are effective and reliable. The precision of armature position estimation compared with the precision of the laser displacement sensor is relatively limited. At the same time, there are some unmodeled factors and random disturbances in control system. Meanwhile, there is error in the testing process of current change rate. All the above makes the current change rate of the position estimation point be still within a certain range fluctuation after current control convergence, and the real position of the position estimation point also fluctuates within a certain range. Besides, the control system works stably, which verifies the feasibility and stability of the sensorless landing control strategy.

In order to explore the operation condition of BPMA in a working cycle under sensorless landing control, Figure 13 shows the current and displacement in the cycle without landing control, the second cycle (in iteration), and 200th cycle (complete converged), respectively.

The current matches well in the positive excitation stage, which proves the effectiveness of the current controller. The landing current with initial positive value gradually decreases with the iteration, until it is negative value after the iteration converged. Compared with the cycle without landing control, the response time of BPMA increases by $6.8 \%$ when the iteration converged, while the landing current changes from $13 \mathrm{~A}$ to $-5.7 \mathrm{~A}$ and the electromagnetic force decreases by $73.5 \%$, which effectively reduces the impact of landing. This indicates that the landing control has a negative effect on the fast response of the actuator.

In order to further analyze the performance indicators of landing, the local detail view of velocity and displacement in different conditions are shown in Figure 14. The landing velocity decreases by $52.1 \%$ when the iteration converged, while the first bounce decreases by $85.7 \%$ and the second bounce is avoided. The landing speed in the $200^{\text {th }}$ working cycle is $3.2 \mathrm{~m} / \mathrm{s}$, which match the design target landing velocity.

The motion curve and landing velocity of each working cycle after iteration convergence are also different, because of unmodeled factors and random disturbances in control system. For more comprehensive analysis of the continuous operating performance, the percentage of landing velocity distribution with or without the sensorless landing control strategy is calculated as shown in Figure 15. Figure 16 

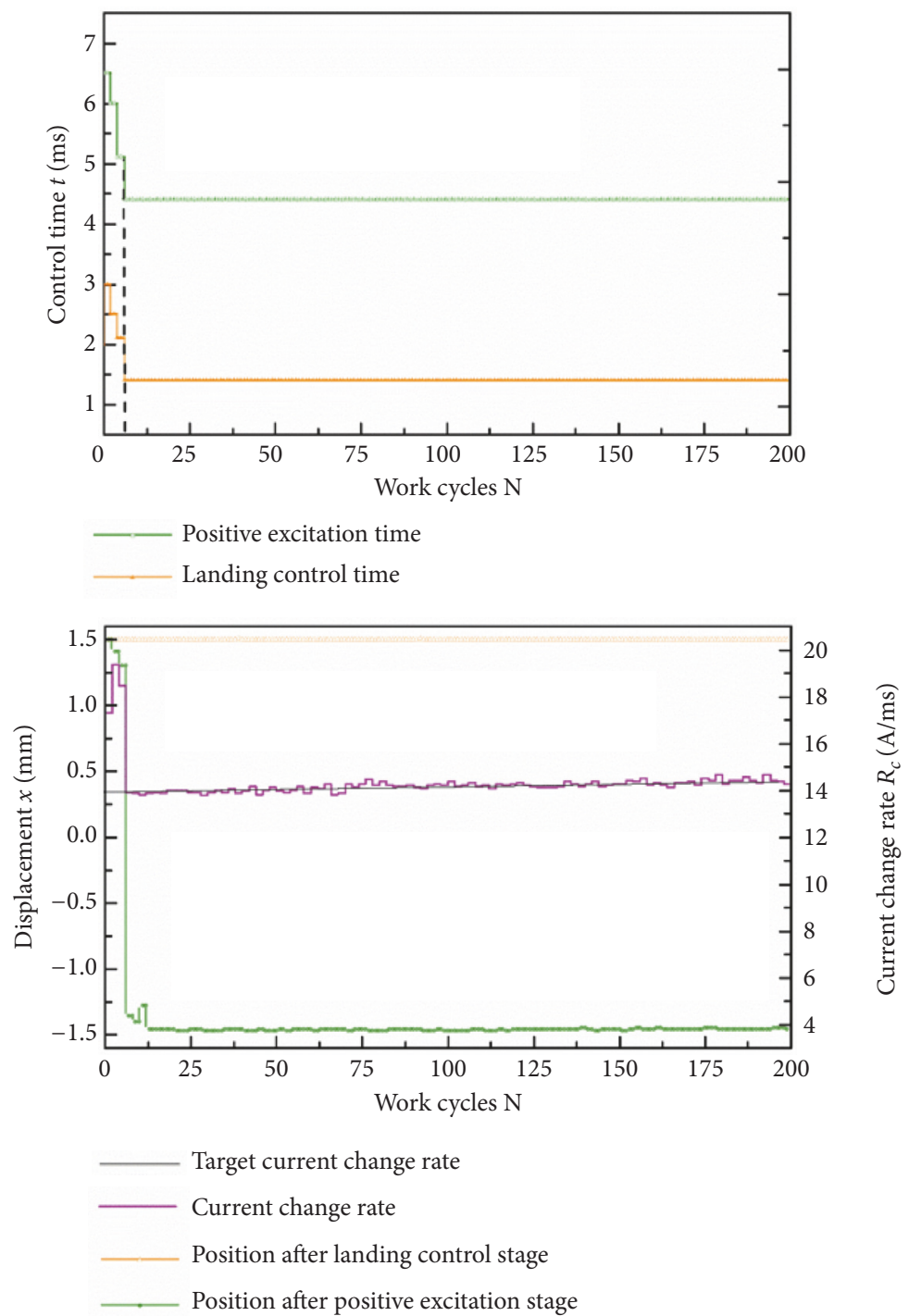

FIGURE 12: 200 cycles successional test under the proposed sensorless landing control.

calculates the sound intensity of BPMA at different working frequency with or without the proposed landing control strategy.

The landing velocity with and without the landing control focuses on $3.0 \pm 0.2 \mathrm{~m} / \mathrm{s}$ and $6.2 \pm 0.3 \mathrm{~m} / \mathrm{s}$, respectively. The landing velocity decreased more than $50 \%$ according sensorless landing control. The undesired impact issues of noise, vibration, and harshness is alleviated effectively. The sound intensity of BPMA at different frequencies after landing control is reduced more than $4 \mathrm{~dB}$. The proposed sensorless landing control strategy effectively improves the operation quality of BPMA.

\section{Conclusion}

A new sensorless landing control strategy was proposed using armature position detection instead of real-time displacement estimation or displacement sensor, combined with the landing control goal and sensorless technology features of the
BPMA. The feasibility and effectiveness of the method are verified by both experiment and simulation.

The first bounce decreases by $85.7 \%$ and the second bounce is avoided when the iteration converged, while the response time increased by $6.8 \%$. Meanwhile, the landing velocity decreased more than $50 \%$ and sound intensity at different frequencies reduced more than $4 \mathrm{~dB}$ with sensorless landing control. The proposed sensorless landing control strategy effectively improves the operation quality of BPMA.

\section{Data Availability}

The data used to support the findings of this study are available from the corresponding author upon request.

\section{Conflicts of Interest}

The authors declare that they have no conflicts of interest. 

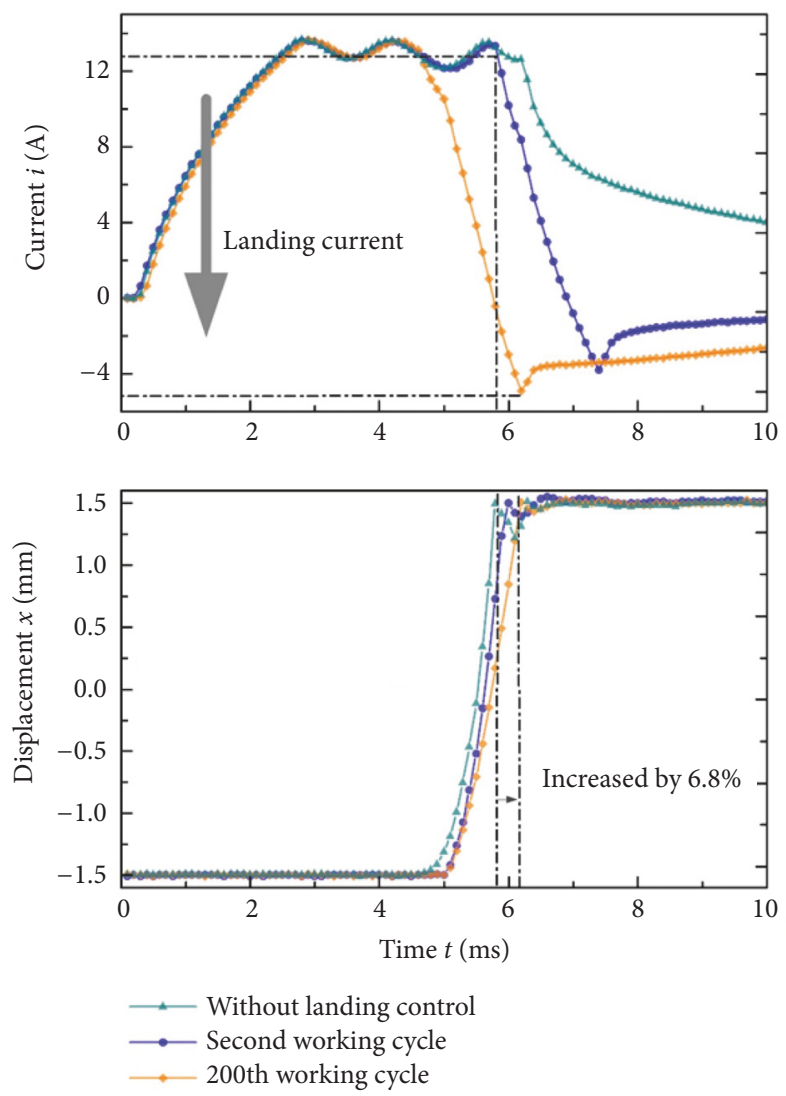

FIGURE 13: Current and displacement in different conditions.
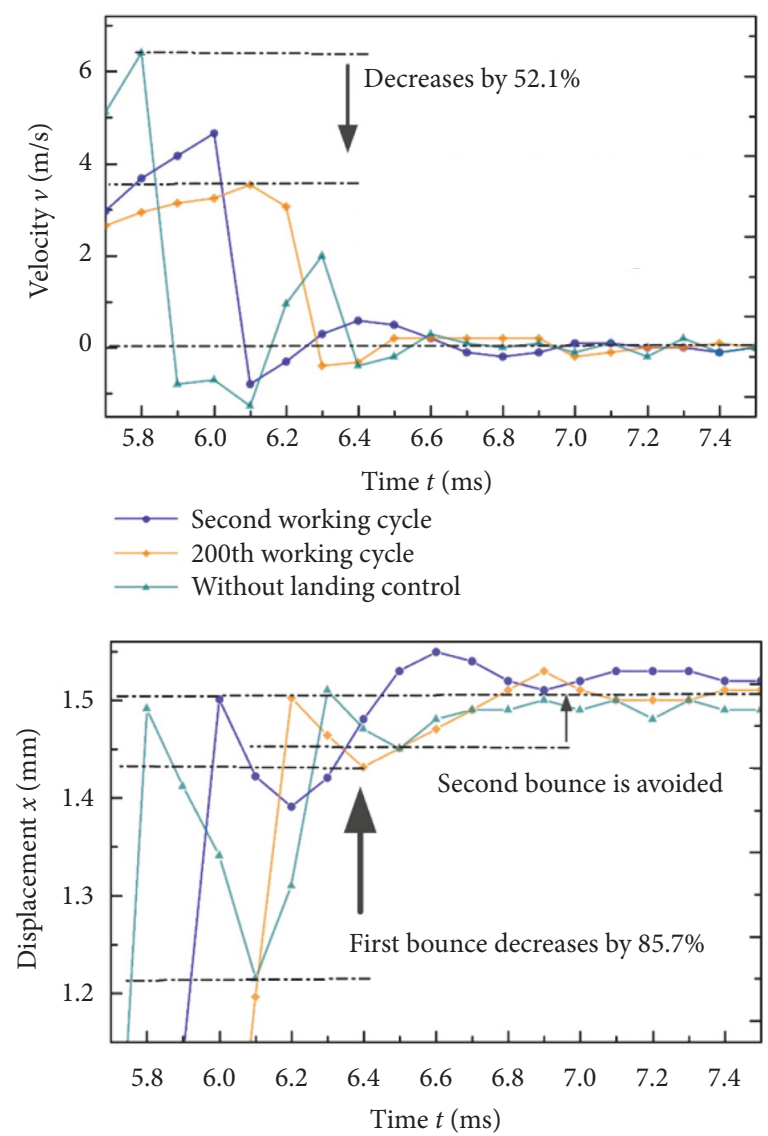

FIGURE 14: Local detail view of velocity and displacement in different conditions. 


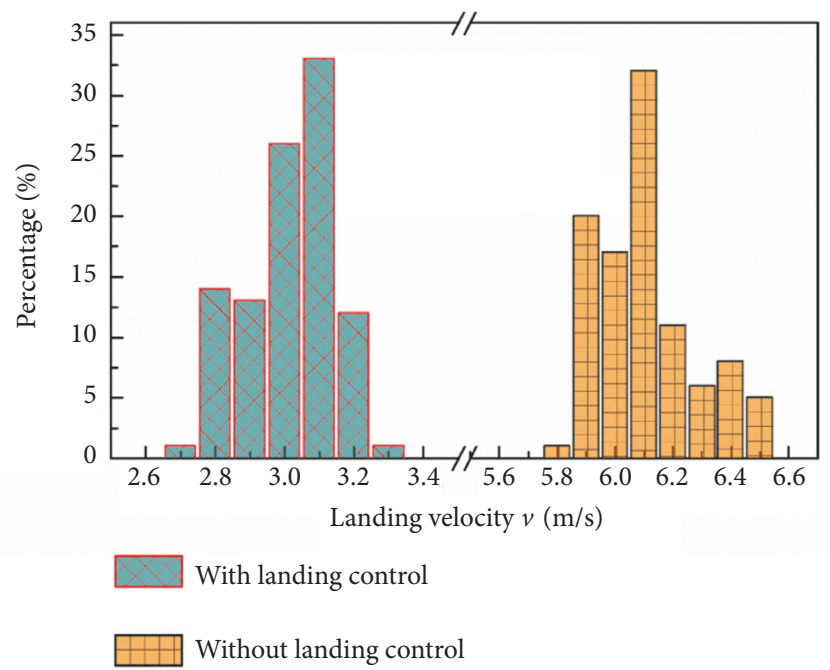

FIGURE 15: Statistical result of landing velocity.

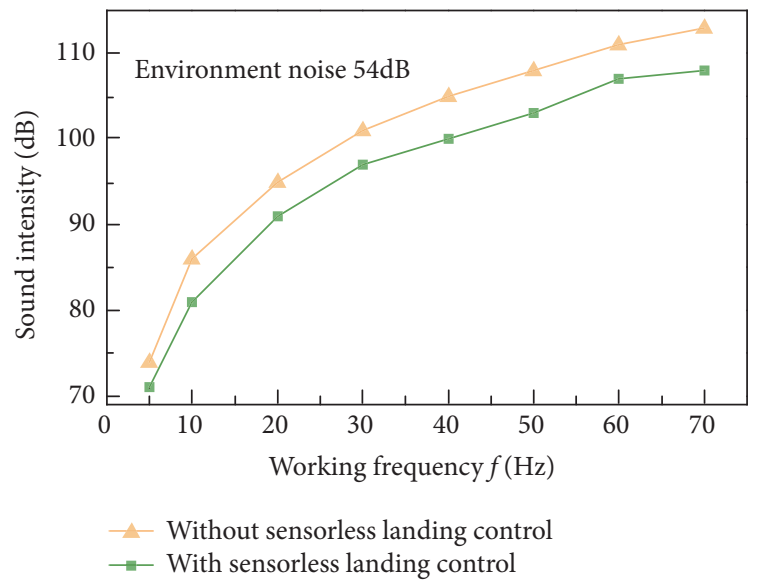

FIGURE 16: Sound intensity of BPMA at different working frequency.

\section{Authors' Contributions}

Bo Li and Cao Tan contributed to the conceiving, designing, planning the study, and commenting on the manuscript. Geng Wang contributed to data analysis and writing the manuscript. Wenqing Ge and Binbin Sun contributed to editing of the manuscript and approving the published version.

\section{Acknowledgments}

This research was funded by the National Natural Science Foundation of China [51875326]; National Natural Science Foundation of China [51805301]; National Key Research and Development Project, China [2017YFB0102004]; Shandong Provincial Agricultural Equipment Innovation Project, China [NJGG201505].

\section{References}

[1] C. Tan, W. Ge, X. Fan, J. Lu, B. Li, and B. Sun, "Bi-stable actuator measurement method based on voice coil motor," Sensors and Actuators A: Physical, vol. 285, pp. 59-66, 2019.

[2] J. Kim and D. K. Lieu, "A new electromagnetic engine valve actuator with less energy consumption for variable valve timing," Journal of Mechanical Science and Technology, vol. 21, no. 4, pp. 602-606, 2007.

[3] K. Pancharoen, D. Zhu, and S. P. Beeby, “Temperature dependence of a magnetically levitated electromagnetic vibration energy harvester," Sensors and Actuators A: Physical, vol. 256, pp. 1-11, 2017.

[4] X. Wang, H. Lin, S. L. Ho, S. Fang, and P. Jin, "Analysis of dynamic characteristics of permanent magnet contactor with sensorless displacement profile control," IEEE Transactions on Magnetics, vol. 46, no. 6, pp. 1633-1636, 2010.

[5] C. Tan, B. Li, W. Ge, and B. Sun, "Design and analysis of a bistable linear force actuator for directly-driven metering pump," Smart Materials and Structures, vol. 27, no. 10, Article ID 107001, 2018.

[6] M. Montazeri-Gh, M. Nasiri, M. Rajabi, and M. Jamshidfard, "Actuator-based hardware-in-the-loop testing of a jet engine fuel control unit in flight conditions," Simulation Modelling Practice and Theory, vol. 21, no. 1, pp. 65-77, 2012.

[7] Y. L. Yin, P. Sun, L. I. Shu-Ling et al., "Study on control method and dynamic performance of the bistable solenoid valve," Machinery Design \& Manufacture, vol. 8, pp. 143-147, 2014.

[8] J. Lee, E. M. Dede, D. Banerjee, and H. Iizuka, "Magnetic force enhancement in a linear actuator by air-gap magnetic field distribution optimization and design," Finite Elements in Analysis and Design, vol. 58, pp. 44-52, 2012.

[9] I. Yatchev, K. Hinov, and N. Trifonov, "Characteristics of a bistable permanent magnet linear actuator with soft magnetic mover," International Journal of Applied Electromagnetics and Mechanics, vol. 27, no. 1-2, pp. 43-52, 2008. 
[10] J. You, K. Zhang, Z. Zhu, and H. Liang, "Novel design and research for a high-retaining-force, bi-directional, electromagnetic valve actuator with double-layer permanent magnets," Journal of Magnetics, vol. 21, no. 1, pp. 65-71, 2016.

[11] C. Tan, S. Chang, and L. Liu, "Hierarchical genetic-particle swarm optimization for Bi-stable permanent magnet actuator," Applied Soft Computing, vol. 61, pp. 1-7, 2017.

[12] L. Liu and S. Chang, "Motion control of an electromagnetic valve actuator based on the inverse system method," Proceedings of the Institution of Mechanical Engineers, Part D: Journal of Automobile Engineering, vol. 226, no. 8, pp. 85-93, 2011.

[13] P. Mercorelli, "An antisaturating adaptive preaction and a slide surface to achieve soft landing control for electromagnetic actuators," IEEE/ASME Transactions on Mechatronics, vol. 17, no. 1, pp. 76-85, 2012.

[14] Y.-P. Yang, J.-J. Liu, D.-H. Ye, Y.-R. Chen, and P.-H. Lu, "Multiobjective optimal design and soft landing control of an electromagnetic valve actuator for a camless engine," IEEE/ASME Transactions on Mechatronics, vol. 18, no. 3, pp. 963-972, 2013.

[15] A. G. Espinosa, J.-R. R. Ruiz, and X. A. Morera, "A sensorless method for controlling the closure of a contactor," IEEE Transactions on Magnetics, vol. 43, no. 10, pp. 3896-3903, 2007.

[16] J. Tsai, C. R. Koch, and M. Saif, "Cycle adaptive feedforward approach controllers for an electromagnetic valve actuator," IEEE Transactions on Control Systems Technology, vol. 20, no. 3, pp. 738-746, 2012.

[17] E. O. Ranft, G. Van Schoor, and C. P. D. Rand, "Self-sensing for electromagnetic actuators. Part II: Position estimation," Sensors and Actuators A: Physical, vol. 172, no. 2, pp. 410-419, 2011.

[18] I. Dülk and D. Kovácsházy, "A sensorless method for detecting spool position in solenoid actuators," Carpathian Journal of electronic and computer engineering, vol. 6, no. 1, pp. 36-43, 2013.

[19] S. Nagai, T. Nozaki, and A. Kawamura, "Environmental robust position control for compact solenoid actuators by sensorless simultaneous estimation of position and force," IEEE Transactions on Industrial Electronics, vol. 63, no. 8, pp. 5078-5086, 2013.

[20] A. Sadighi and W.-J. Kim, "Adaptive-neuro-fuzzy-based sensorless control of a smart-material actuator," IEEE/ASME Transactions on Mechatronics, vol. 16, no. 2, pp. 371-379, 2011.

[21] S. Nagai, T. Nozaki, and A. Kawamura, "Real-time sensorless estimation of position and force for solenoid actuators," IEEJ Journal of Industry Applications, vol. 5, no. 2, pp. 32-38, 2016.

[22] P. Mercorelli, "An adaptive and optimized switching observer for sensorless control of an electromagnetic valve actuator in camless internal combustion engines," Asian Journal of Control, vol. 16, no. 4, pp. 959-973, 2014.

[23] P. Mercorelli, "A hysteresis hybrid extended Kalman filter as an observer for sensorless valve control in camless internal combustion engines," IEEE Transactions on Industry Applications, vol. 48, no. 6, pp. 1940-1949, 2012.

[24] P. Mercorelli, "A two-stage augmented extended Kalman filter as an observer for sensorless valve control in camless internal combustion engines," IEEE Transactions on Industrial Electronics, vol. 59, no. 11, pp. 4236-4247, 2012. 


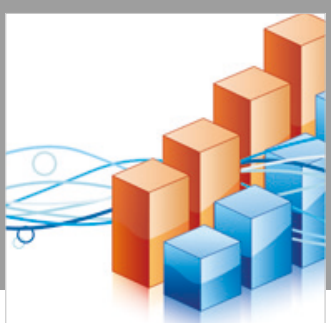

Advances in

Operations Research

\section{-n-m}
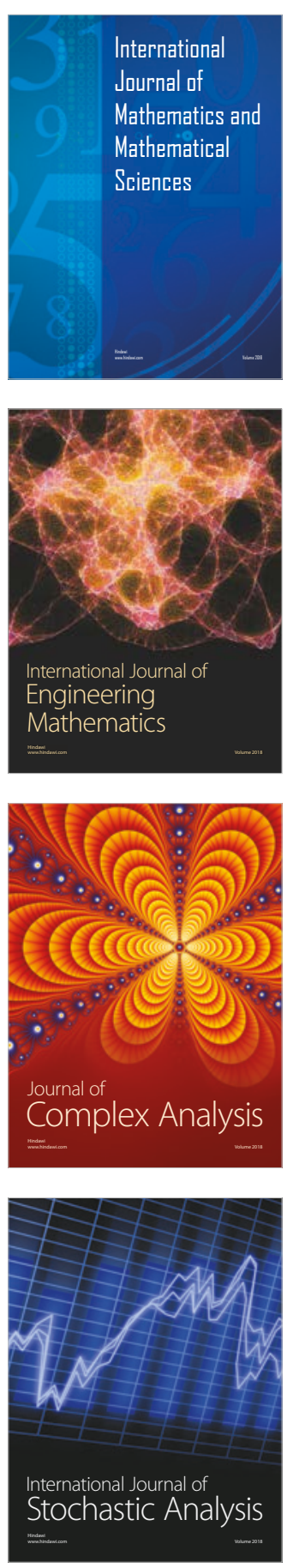
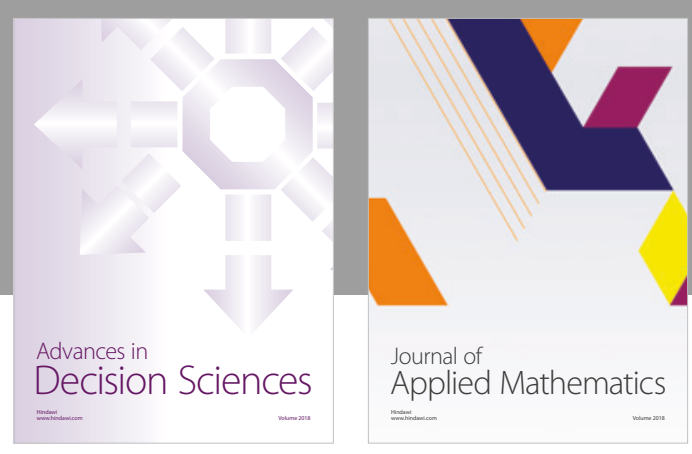

Journal of

Applied Mathematics
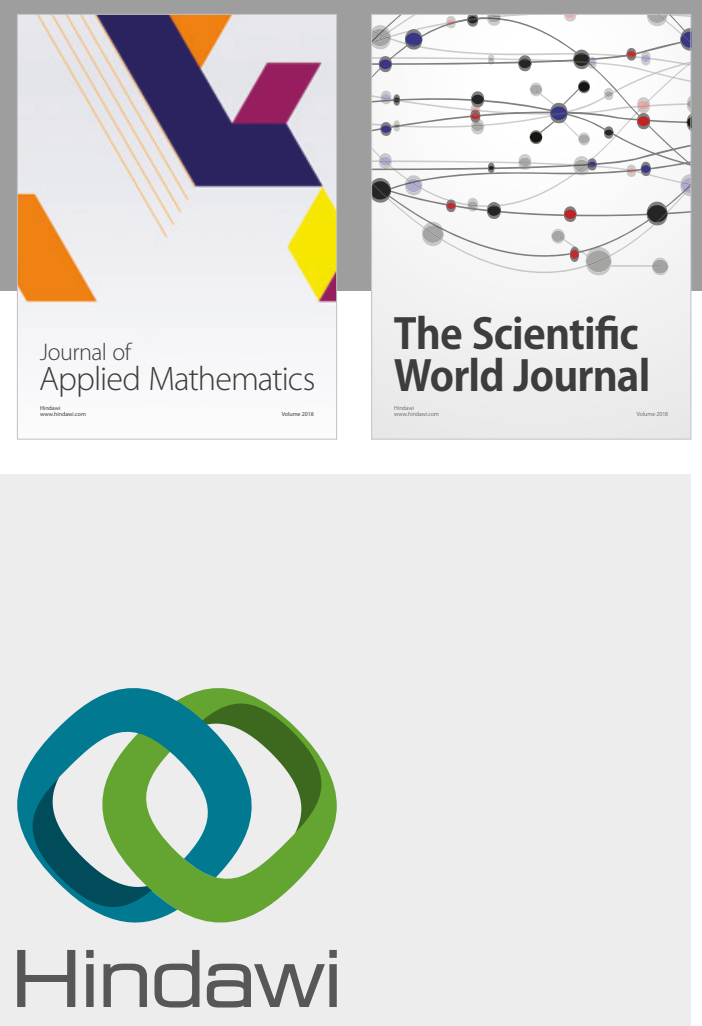

Submit your manuscripts at

www.hindawi.com

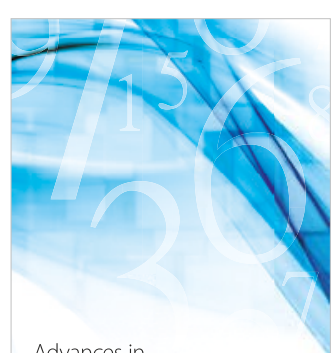

Advances in
Numerical Analysis
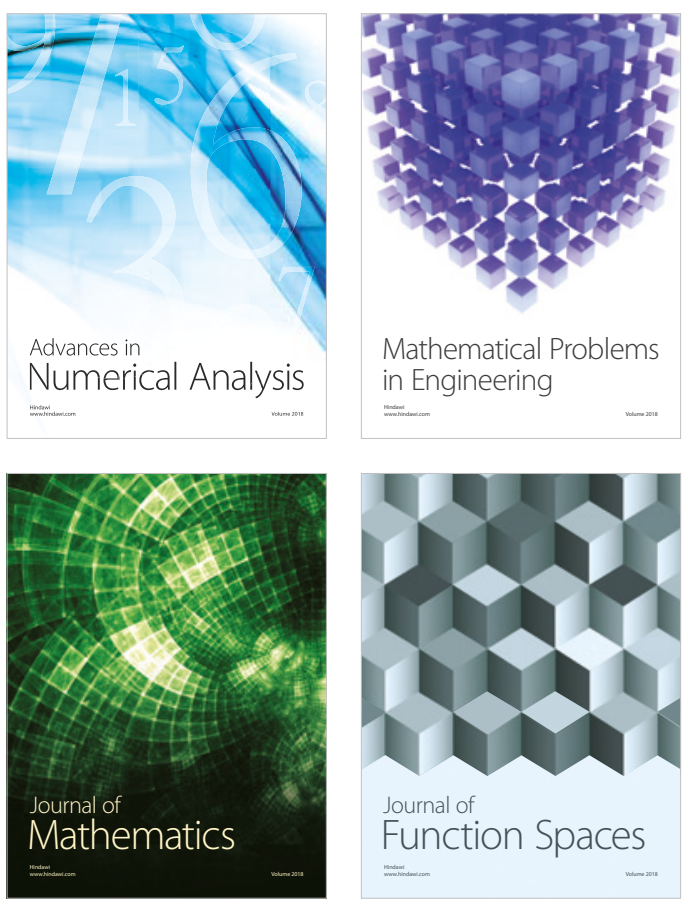

Mathematical Problems in Engineering

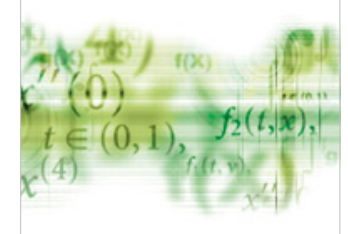

International Journal of

Differential Equations

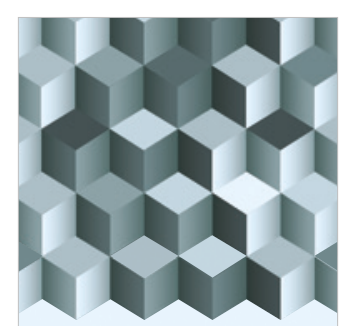

Journal of

Function Spaces

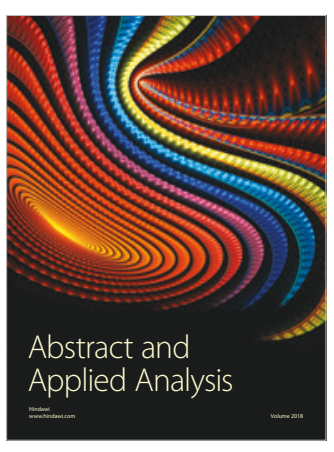

The Scientific

World Journal

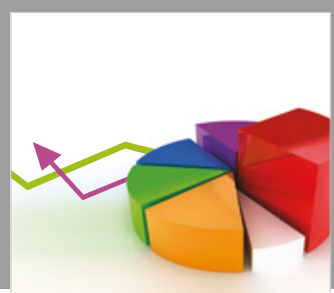

Journal of

Probability and Statistics
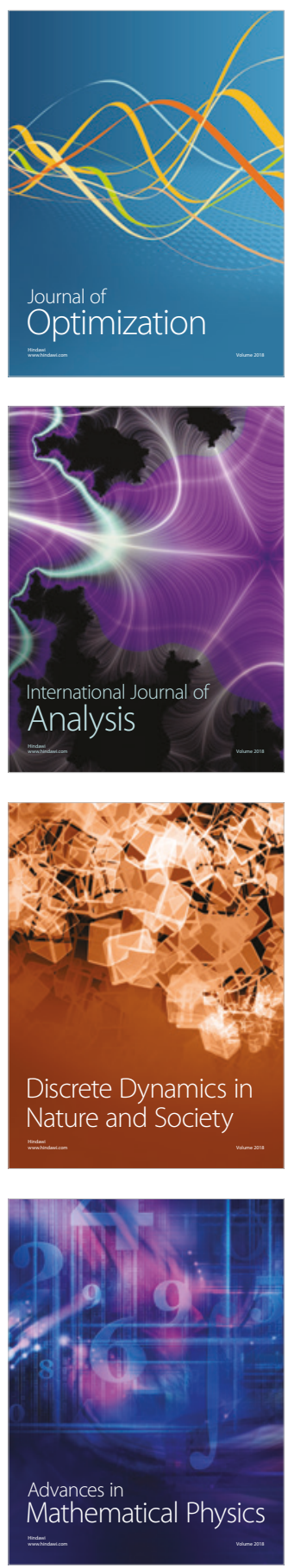